\author{
for \\ the \\ communication \\ entitled
}

\title{
Chiral Lewis Acid Catalyzed Highly Enantioselective [4 + 3] Cycloaddition Reactions of Nitrogen-Stabilized Oxyallyl Cations Derived from Allenamides.
}

authored by

Jian Huang and Richard P. Hsung*

Department of Chemistry, University of Minnesota, Minneapolis, MN 55455 


\section{General Methods.}

Unless stated otherwise, reactions were performed in flame-dried glassware under a positive pressure of $\mathrm{N}_{2}$ using freshly distilled solvents. Enantiomeric excess was determined using HPLC on a Chiralcel-OD chiral column) with a UV detector.

\section{Procedures for Generating Chiral Catalysts.}

\section{Cobalt (III)-Salen Complex.}

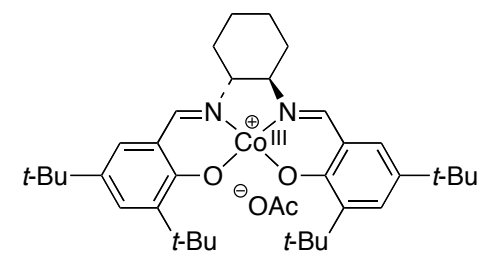

To a stirred solution of salen ligand $11 \mathrm{in} \mathrm{MeOH}(0.1 M)$, was added dropwise $\mathrm{Co}(\mathrm{OAc})_{2} \bullet 4 \mathrm{H}_{2} \mathrm{O}(1$ equiv, $0.2 \mathrm{M}$ in $\mathrm{MeOH}$ ). The mixture was refluxed for $1 \mathrm{~h}$. After cooling to $\mathrm{rt}$, the crystal was collected by filtration, and it was washed with ice-cold $\mathrm{MeOH}$ and dried under reduced pressure.

\section{Activated Oxazaborolidine Complex.}

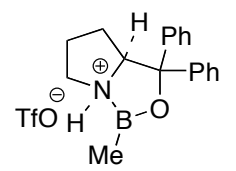

To an aliquot of the oxazaborolidine $12(0.030 \mathrm{~mL}, 0.030 \mathrm{mmol})$ in $2 \mathrm{~mL}$ of $\mathrm{CH}_{2} \mathrm{Cl}_{2}$ at $-78{ }^{\circ} \mathrm{C}$ was added dropwise $125.0 \mu \mathrm{L}$ of HOTf $\left[0.20 \mathrm{M}\right.$ solution in $\left.\mathrm{CH}_{2} \mathrm{Cl}_{2}\right]$. After $15 \mathrm{~min}$ at $-78{ }^{\circ} \mathrm{C}$, a colorless homogeneous solution was obtained which was ready to use.

\section{General Procedure to Preparations of All Other Chiral Catalysts.}

To a solution of a respective Lewis acid in acetone $(0.003 \mathrm{M})$ at $\mathrm{rt}$ was added 1.2 equiv of a respective chiral ligand. After $5 \mathrm{~h}$, it was ready to use.

\section{Preparation of $\mathrm{Cu}(\mathrm{II})$ Complex of Bisoxazoline Ligand $15 \mathrm{~b}$ with Antimonate as the Counter Anion.}

To a dry flask in an inert atmosphere dry box was added $4.10 \mathrm{mg}(0.030 \mathrm{mmol}) \mathrm{CuCl}_{2}, 20.9 \mathrm{mg}(0.060$ $\mathrm{mmol}) \mathrm{AgSbF}_{6}$, and $17.5 \mathrm{mg}(0.036 \mathrm{mmol})$ ligand $15 \mathrm{~b}$. The flask was fitted with a septum, and was removed from the dry box and charged with $1.5 \mathrm{~mL}$ of $\mathrm{CH}_{2} \mathrm{Cl}_{2}$. The resulting heterogeneous mixture was stirred for $12 \mathrm{~h}$ in the absence of light before being filtered through celite into a dry flask. 


\section{General Procedures for Catalytic Asymmetric [4 + 3] Cycloaddition Reactions.}

\section{General Procedure for Preparation of DMDO.}

To a 1-L three-neck RBF equipped with a sturdy stirring bar were added $100 \mathrm{~mL}$ of distilled $\mathrm{H}_{2} \mathrm{O}, 80$ $\mathrm{mL}$ of acetone, and $24.0 \mathrm{~g}$ of $\mathrm{NaHCO}_{3}$. The resulting mixture was vigorously stirred while being cooled in an ice bath for $5 \mathrm{~min}$. To this mixture was added $50.0 \mathrm{~g}$ of $\mathrm{Oxone}^{\mathrm{TM}}$ in two consecutive portions within $10 \mathrm{~min}$. The mixture was stirred at $\mathrm{rt}$ for an additional 15-20 min. DMDO was then distilled over as a solution in acetone under house vacuum $(\sim 50-120 \mathrm{~mm} \mathrm{Hg})$. The distillation process took about 1-2 $\mathrm{h}$. The DMDO solution was collected at $-78{ }^{\circ} \mathrm{C}$ and dried over by $4 \AA$ molecular sieve. Concentration of the DMDO solution was determined using the standard thioanisole NMR-titration protocol.

To a solution of an appropriate catalytic system in acetone at $-78{ }^{\circ} \mathrm{C}$ were added an appropriate allenamide [15.0 mg, $0.12 \mathrm{mmol}$ ], diene [9.0 equiv, $1.08 \mathrm{mmol}$ ], and $300.0 \mathrm{mg} 4 \AA$ molecular sieve. After stirring at $-78{ }^{\circ} \mathrm{C}$ for $15 \mathrm{~min}, 3.0-5.0$ equiv of DMDO were added over $3-4 \mathrm{~h}$ at $-78{ }^{\circ} \mathrm{C}$ as a chilled solution in acetone using a syringe pump. The syringe pump was cooled using dry ice at all time during the addition. After the addition, the reaction mixture was stirred for another $4 \mathrm{~h}$ before being concentrated in vacuo. The crude residue was purified via silica gel column chromatography (gradient eluent: $0 \%$ to $60 \%$ ethyl acetate in hexane).

\section{Preparation OF (FURAN-3-YL-METHOXY)-TRIPHENYL-SILANE.}

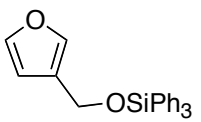

To a stirring solution of 3-hydroxymethyl furan (1.00 g, $10.2 \mathrm{mmol})$ in $\mathrm{CH}_{2} \mathrm{Cl}_{2}(15 \mathrm{~mL})$ were added imidazole $(1.04 \mathrm{~g}, 15.3 \mathrm{mmol})$ and triphenylsilylchloride $(3.60 \mathrm{~g}, 12.2 \mathrm{mmol})$ at $0{ }^{\circ} \mathrm{C}$. The mixture was allowed to warm up to rt and stirred for $10 \mathrm{~h}$. After completion, $10 \mathrm{~mL}$ sat aq $\mathrm{NaHCO}_{3}$ was added, and the aqueous solution was extracted with $\mathrm{CH}_{2} \mathrm{Cl}_{2}(3 \mathrm{X} 25 \mathrm{~mL})$. The combined organic extracts were washed with sat aq $\mathrm{NaCl}$, dried over by anhy $\mathrm{Na}_{2} \mathrm{SO}_{4}$, filtered, and concentrated in vacuo. The crude residue was purified via silica gel column chromatography (using EtOAc/hexane as eluent) to give the desired furan derivative in $89 \%$ yield. $R_{f}=0.55[25 \%$ EtOAc in hexane];

${ }^{1} \mathrm{H}$ NMR $\left(500 \mathrm{MHz}, \mathrm{CDCl}_{3}\right) \delta 4.75(\mathrm{~d}, 2 \mathrm{H}, J=2.0 \mathrm{~Hz}), 6.31(\mathrm{dd}, 1 \mathrm{H}, J=1.0,2.0 \mathrm{~Hz}), 7.30-7.61(\mathrm{~m}, 11 \mathrm{H})$, 7.73-7.82 (m, 6H); ${ }^{13} \mathrm{C}$ NMR (125 MHz, $\left.\mathrm{CDCl}_{3}\right) \delta$ 57.9, 109.7, 127.9, 129.7, 130.1, 133.9, 135.4, 139.7, 143.0; IR (thin film) $\mathrm{cm}^{-1} 2959 \mathrm{w}, 2917 \mathrm{~m}, 1752 \mathrm{~s}, 1722 \mathrm{~s}, 1427 \mathrm{~s}$; mass spectrum (EI): $\mathrm{m} / \mathrm{e}$ (\% relative intensity) 235 (29) $\mathrm{M}^{+}, 259$ (16), 199 (100), 173 (20). 


\section{Preparation of the Chiral Ester for X-ray Analysis.}

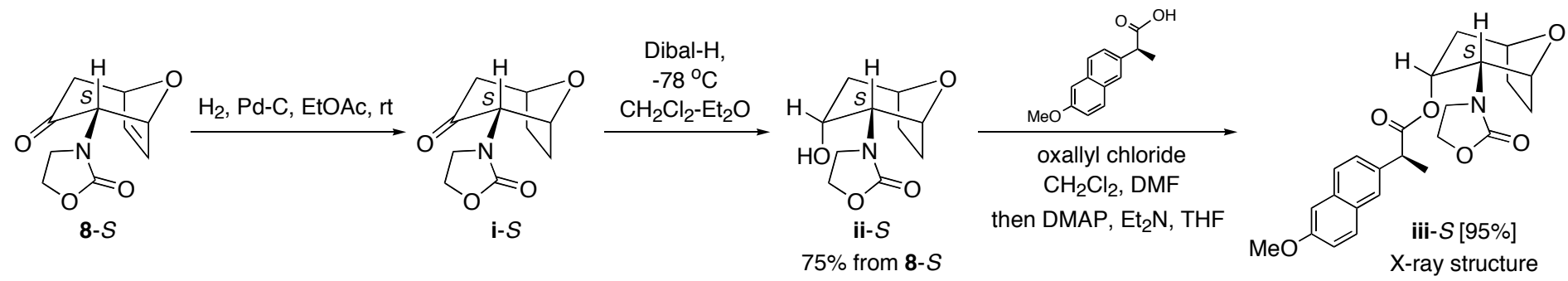

\section{Hydrogenation.}

To a solution of $20.0 \mathrm{mg}(0.096 \mathrm{mmol})$ of $8-S$ in $4 \mathrm{~mL}$ EtOAc at $\mathrm{rt}$ was added $5 \% \mathrm{Pd} / \mathrm{C}(5-10 \mathrm{mg})$. The heterogeneous mixture was stirred under 1 atm of $\mathrm{H}_{2}$ for $3 \mathrm{~h}$. Filtration through Celite led to quantitative isolation of the desired hydrogenated product $\mathbf{i}-S$.

$\mathrm{R}_{\mathrm{f}}=0.43\left[\right.$ EtOAc]; mp $76.5-79.3{ }^{\circ} \mathrm{C}$;

${ }^{1} \mathrm{H}$ NMR $\left(500 \mathrm{MHz}, \mathrm{CDCl}_{3}\right) \delta$ 1.76-1.89 (m, 2H), 2.09-2.14 (m, 2H), $2.40(\mathrm{~d}, 1 \mathrm{H}, \mathrm{J}=15.5 \mathrm{~Hz}), 2.81(\mathrm{dd}, 1 \mathrm{H}, \mathrm{J}$ $=1.0,7.5,7.5 \mathrm{~Hz}), 3.55(\mathrm{ddd}, 1 \mathrm{H}, \mathrm{J}=7.0,9.0,9.0 \mathrm{~Hz}), 4.02(\mathrm{ddd}, 1 \mathrm{H}, \mathrm{J}=7.0,9.0,9.0 \mathrm{~Hz}), 4.35$ (ddd, $1 \mathrm{H}, \mathrm{J}=$ 7.0, 9.0, 9.0 Hz), $4.43(\mathrm{ddd}, 1 \mathrm{H}, \mathrm{J}=7.0,9.0,9.0 \mathrm{~Hz}), 4.70-4.74(\mathrm{~m}, 1 \mathrm{H}), 4.76-4.80(\mathrm{~m}, 2 \mathrm{H})$; ${ }^{13} \mathrm{C} \mathrm{NMR}(125$ $\mathrm{MHz}_{\mathrm{CDCl}}$ ) $\delta 26.9,29.8,42.7,48.8,62.7,65.0,75.7,77.7,158.7,202.9 ;$ IR (thin film) $\mathrm{cm}^{-1} 2991 \mathrm{~s}, 2959 \mathrm{~s}$, 2921s, 1730s, 1712s; mass spectrum (ESI): m/e (\% relative intensity) $212(100)(\mathrm{M}+1)^{+}, 197(76), 166(28)$, 122 (19), 107 (96).

\section{Dibal-H Reduction.}

To a stirring solution of $\mathbf{i}-S(20.0 \mathrm{mg}, 0.096 \mathrm{mmol})$ in $3 \mathrm{~mL}$ of ether/ $\mathrm{CH}_{2} \mathrm{Cl}_{2}$ (ratio: $\left.5: 1\right)$ at $-78^{\circ} \mathrm{C}$ was added DIBAL-H $(0.33 \mathrm{~mL}, 1.0 \mathrm{M}$ in toluene). The reaction was stirred at this temperature for $1 \mathrm{~h}$ before being warmed up to rt over $2 \mathrm{~h}$, The reaction mixture was then quenched by sat aq $\mathrm{NH}_{4} \mathrm{Cl}$, and the aqueous layer was separated and extracted with ether ( 3 X $25 \mathrm{~mL}$ ). The combined organic extracts were washed with sat aq $\mathrm{NaCl}$, dried over by anhy $\mathrm{Na}_{2} \mathrm{SO}_{4}$, filtered, and concentrated in vacuo. The crude residue was purified via silica gel column chromatography (using EtOAc/hexane as eluent) to give the desired alcohol ii-S in $75 \%$ yield.

$R_{f}=0.15[\mathrm{EtOAc}]$

${ }^{1} \mathrm{H}$ NMR $\left(500 \mathrm{MHz}, \mathrm{CDCl}_{3}\right) \delta 1.76(\mathrm{ddd}, 1 \mathrm{H}, J=1.5,1.5,14.5 \mathrm{~Hz}), 1.93(\mathrm{~m}, 2 \mathrm{H}), 2.11(\mathrm{ddd}, 1 \mathrm{H}, J=4.5,4.5$, $14.5 \mathrm{~Hz}), 2.24(\mathrm{~m}, 1 \mathrm{H}), 2.56(\mathrm{~m}, 1 \mathrm{H}), 3.24(\mathrm{br}, 1 \mathrm{H}), 3.66(\mathrm{ddd}, 1 \mathrm{H}, J=1.0,7.5,16.5 \mathrm{~Hz}), 3.86(\mathrm{dd}, 1 \mathrm{H}, J=4.5$, $4.5 \mathrm{~Hz}), 4.03(\mathrm{ddd}, 1 \mathrm{H}, J=7.5,9.0,9.0 \mathrm{~Hz}), 4.33(\mathrm{~m}, 5 \mathrm{H}) ;{ }^{13} \mathrm{C} \mathrm{NMR}\left(125 \mathrm{MHz}, \mathrm{CDCl}_{3}\right) \delta 26.8,29.0,38.6$, 45.0, 57.5, 62.6, 67.3, 74.3, 75.1, 159.0; IR (thin film) $\mathrm{cm}^{-1} 3425 \mathrm{br}, 2944 \mathrm{~s}, 1728 \mathrm{~s}, 1484 \mathrm{~s}$; mass spectrum (APCI): m/e (\% relative intensity) $214(100)(\mathrm{M}+1)^{+}, 196(24), 170(26), 138$ (12). 


\section{Esterification.}

To a stirred solution of ii- $S$ obtained above in $3 \mathrm{~mL}$ THF at rt was added DMAP (3.0 mg), Et ${ }_{3} \mathrm{~N}(0.05$ $\mathrm{mL}$ ), and $(S)-(+)-6$-methoxy- $\alpha$-methyl-2-naphthaleneacetic Chloride (70.0 mg, $0.28 \mathrm{mmol})$. The reaction mixture was stirred at $\mathrm{rt}$ for $10 \mathrm{~h}$ before being quenched by sat aq $\mathrm{NaHCO}_{3}$. The aqueous layer was separated and extracted with EtOAc ( 3 X $25 \mathrm{~mL})$. The combined organic extracts were washed with sat aq $\mathrm{NaCl}$, dried over by anhy $\mathrm{Na}_{2} \mathrm{SO}_{4}$, filtered, and concentrated in vacuo. The crude residue was purified via silica gel column chromatography [using EtOAc/hexane as eluent] to give the desired ester iii- $S$ in $95 \%$ yield. The ester iii- $S$ as recrystallized from ether/ $\mathrm{CH}_{2} \mathrm{Cl}_{2} /$ petane 3 times to get X-ray quality single crystals.

$R_{f}=0.51[\mathrm{EtOAc}] ; \operatorname{mp} 117.3-122.2^{\circ} \mathrm{C} ;[\alpha]_{\mathrm{D}}^{23}=-53.3\left(\mathrm{c}=0.63, \mathrm{CH}_{2} \mathrm{Cl}_{2}\right)$;

${ }^{1} \mathrm{H}$ NMR (500 MHz, $\left.\mathrm{CDCl}_{3}\right) \delta 1.37(\mathrm{~m}, 1 \mathrm{H}), 1.65(\mathrm{~m}, 5 \mathrm{H}), 1.83(\mathrm{~m}, 1 \mathrm{H}), 2.12(\mathrm{~m}, 2 \mathrm{H}), 3.08(\mathrm{ddd}, 1 \mathrm{H}, J=1.0$, 7.5, 7.5 Hz), $3.24(\mathrm{ddd}, 1 \mathrm{H}, J=1.0,7.5,7.5 \mathrm{~Hz}), 3.78(\mathrm{dd}, 1 \mathrm{H}, J=7.5,15.0 \mathrm{~Hz}), 3.88(\mathrm{dd}, 1 \mathrm{H}, J=7.5,15.0$ Hz), 3.93 (m, 4H), $4.13(\mathrm{dd}, 1 \mathrm{H}, J=4.0,4.0 \mathrm{~Hz}), 4.25(\mathrm{~m}, 2 \mathrm{H}), 5.28(\mathrm{~m}, 1 \mathrm{H}), 7.12(\mathrm{~m}, 1 \mathrm{H}), 7.17(\mathrm{~m}, 1 \mathrm{H}), 7.38$ (m, 1H), $7.70(\mathrm{~m}, 3 \mathrm{H}) ;{ }^{13} \mathrm{C} \mathrm{NMR}\left(125 \mathrm{MHz}, \mathrm{CDCl}_{3}\right) \delta$ 17.3, 26.7, 28.4, 35.5, 43.8, 46.1, 55.0, 55.3, 62.0, 71.0, $73.4,74.9,105.5,119.5,125.8,127.5,128.8,129.1,133.8,134.6,157.9,173.2$ (missing 2 carbons due to overlap); IR (thin film) $\mathrm{cm}^{-1} 3054 \mathrm{~m}, 2954 \mathrm{~s}, 2840 \mathrm{~m}, 1734 \mathrm{~s}, 1631 \mathrm{~s}, 1606 \mathrm{~s} ;$ mass spectrum (APCI): m/e (\% relative intensity) 426(100) $(\mathrm{M}+1)^{+}, 214(26), 196(29), 185(51)$.

\section{Characterizations of CyCloadducts.}

\section{Compound 8-S.}

$R_{f}=0.34[\mathrm{EtOAc}] ;[\alpha]_{\mathrm{D}}^{23}=+108.3\left(\mathrm{c}=0.36, \mathrm{CH}_{2} \mathrm{Cl}_{2}\right)$;

${ }^{1} \mathrm{H}$ NMR $\left(500 \mathrm{MHz}, \mathrm{CDCl}_{3}\right) \delta 2.46(\mathrm{~d}, 1 \mathrm{H}, J=16.0 \mathrm{~Hz}), 2.89(\mathrm{dd}, 1 \mathrm{H}, J=5.0,16.0 \mathrm{~Hz}), 3.28(\mathrm{dd}, 1 \mathrm{H}, J=7.5$, $15.0 \mathrm{~Hz}), 3.69$ (ddd, 1H, $J=7.5,15.0 \mathrm{~Hz}), 4.39$ (m, 2H), 4.89 (d, 1H, J = 4.5 Hz), 5.05 (dd, 1H, $J=1.5,4.5$ $\mathrm{Hz}), 5.09(\mathrm{dd}, 1 \mathrm{H}, J=1.5,5.0 \mathrm{~Hz}), 6.28(\mathrm{dd}, 1 \mathrm{H}, J=1.5,6.0 \mathrm{~Hz}), 6.42(\mathrm{dd}, 1 \mathrm{H}, J=1.5,6.0 \mathrm{~Hz}) ;{ }^{13} \mathrm{C} \mathrm{NMR}$ $\left(125 \mathrm{MHz} \mathrm{CDCl}_{3}\right) \delta 42.7,45.7,62.5,65.8,78.0,79.9,131.1,136.0,158.8,200.6$; IR (thin film) $\mathrm{cm}^{-1} 2960 \mathrm{w}$, 2909m, 2850w, 1743m, 1715s; mass spectrum (APCI): m/e (\% relative intensity) $210(39)(\mathrm{M}+1)^{+}, 192(100)$, 166 (15), 148 (16), 123 (37); $t_{r}=21.5 \mathrm{~min}$ (minor), $24.6 \mathrm{~min}$ (major) (20\% $i$-PrOH in hexane).

\section{Compound 18-S.}

$R_{f}=0.48[\mathrm{EtOAc}]$

${ }^{1} \mathrm{H}$ NMR $\left(500 \mathrm{MHz}, \mathrm{CDCl}_{3}\right) \delta 2.05(\mathrm{~d}, 1 \mathrm{H}, J=11.0 \mathrm{~Hz}), 2.28(\mathrm{~m}, 1 \mathrm{H}), 2.42(\mathrm{ddd}, 1 \mathrm{H}, J=3.5,5.0,16.0 \mathrm{~Hz})$, 2.54(ddd, 1H, J = 1.5, 3.5, 16.0 Hz), 2.96 (m, 1H), 3.04 (m, 1H), 3.39 (ddd, 1H, J = 1.5, 2.0, 7.5 Hz), 3.59 (m, $1 \mathrm{H}), 4.35(\mathrm{~m}, 2 \mathrm{H}), 4.68(\mathrm{~d}, 1 \mathrm{H}, J=2.5 \mathrm{~Hz}), 6.01(\mathrm{dd}, 1 \mathrm{H}, J=3.0,6.0 \mathrm{~Hz}), 6.16(\mathrm{~m}, 1 \mathrm{H}) ;{ }^{13} \mathrm{C} \mathrm{NMR}(125 \mathrm{MHz}$, $\left.\mathrm{CDCl}_{3}\right) \delta 39.3,42.7,44.0,45.1,45.2,62.4,66.6,132.7,138.1,159.0,204.0 ;$ IR (thin film) $\mathrm{cm}^{-1} 2960 \mathrm{~m}$, 
2912w, 1733s, 1713s, 1424w; mass spectrum (EI): m/e (\% relative intensity) 207 (100) $\mathrm{M}^{+}, 164(13), 142$ (25), 120 (54), 92 (45); $t_{r}=15.1 \mathrm{~min}$ (minor), $17.5 \mathrm{~min}$ (major) (20\% $i$-PrOH in hexane).

\section{Compound 19-S.}

$R_{f}=0.15[\mathrm{EtOAc}]$

${ }^{1} \mathrm{H}$ NMR (500 MHz, $\left.\mathrm{CDCl}_{3}\right) \delta$ 1.95-2.20 (m, 2H), 2.39-2.45 (m, 3H), $2.85(\mathrm{dd}, 1 \mathrm{H}, J=5.0,16.0 \mathrm{~Hz}), 3.08$ (ddd, $1 \mathrm{H}, J=6.0,8.0,8.0 \mathrm{~Hz}), 3.43(\mathrm{ddd}, 1 \mathrm{H}, J=5.0,8.0,8.0 \mathrm{~Hz}), 4.93(\mathrm{dd}, 1 \mathrm{H}, J=2.0,5.0 \mathrm{~Hz}), 5.06(\mathrm{dd}, 1 \mathrm{H}, J=$ $1.5,5.0 \mathrm{~Hz}), 5.15(\mathrm{~d}, 1 \mathrm{H}, J=5.0 \mathrm{~Hz}), 6.29(\mathrm{dd}, 1 \mathrm{H}, J=2.0,6.0 \mathrm{~Hz}), 6.38(\mathrm{dd}, 1 \mathrm{H}, J=1.56 .0 \mathrm{~Hz})$; ${ }^{13} \mathrm{C} \mathrm{NMR}$ $\left(75 \mathrm{MHz} \mathrm{CDCl}_{3}\right) \delta 18.6,30.2,45.7,45.8,64.3,78.0,80.0,131.7,135.5,176.5,201.3$; IR (thin film) $\mathrm{cm}^{-1}$ 2962s, 2905s, 1717s, 1662s, 1652s, 1418s; mass spectrum (EI): m/e (\% relative intensity) 207 (10) $\mathrm{M}^{+}, 126$ (52), 98 (100), 70 (17), 56 (12); $t_{r}=18.3 \mathrm{~min}$ (minor), $22.5 \mathrm{~min}$ (major) (20\% $i$-PrOH in hexane).

\section{Compound 21-S.}

$R_{f}=0.25$ [EtOAc];

${ }^{1} \mathrm{H}$ NMR $\left(500 \mathrm{MHz}, \mathrm{CDCl}_{3}\right) \delta 1.72-1.92(\mathrm{~m}, 4 \mathrm{H}), 2.41(\mathrm{~d}, 1 \mathrm{H}, J=16.0 \mathrm{~Hz}), 2.46-2.50(\mathrm{~m}, 2 \mathrm{H}), 2.85(\mathrm{dd}, 1 \mathrm{H}, J$ $=5.0,16.0 \mathrm{~Hz}), 2.90-2.96(\mathrm{~m}, 1 \mathrm{H}), 3.20-3.26(\mathrm{~m}, 1 \mathrm{H}), 4.97(\mathrm{dd}, 1 \mathrm{H}, J=2.0,5.0 \mathrm{~Hz}), 5.04(\mathrm{dd}, 1 \mathrm{H}, J=2.0,5.0$ $\mathrm{Hz}), 5.72(\mathrm{~d}, 1 \mathrm{H}, J=5.0 \mathrm{~Hz}), 6.31(\mathrm{dd}, 1 \mathrm{H}, J=2.0,6.0 \mathrm{~Hz}), 6.38(\mathrm{dd}, 1 \mathrm{H}, J=2.0,6.0 \mathrm{~Hz}) ;{ }^{13} \mathrm{C} \mathrm{NMR}(125$ $\left.\mathrm{MHz}_{,} \mathrm{CDCl}_{3}\right) \delta 20.9,23.2,32.0,45.4,46.5,66.5,77.8,79.8,132.4,135.0,144.3,192.4$; IR (thin film) $\mathrm{cm}^{-1}$ 2953s, 2871m, 1720s, 1637s, 1347m; mass spectrum (APCI): m/e (\% relative intensity) $222(100)(\mathrm{M}+1)^{+}, 204$ (11), 176 (29), 140 (12), $100(42) ; t_{r}=15.1 \mathrm{~min}$ (minor), $23.6 \mathrm{~min}$ (major) (20\% $i$-PrOH in hexane).

\section{Compound 22-S (syn).}

$R_{f}=0.41[\mathrm{EtOAc}]$

${ }^{1} \mathrm{H}$ NMR $\left(500 \mathrm{MHz}, \mathrm{CDCl}_{3}\right) \delta 1.53(\mathrm{~s}, 3 \mathrm{H}), 2.44(\mathrm{~d}, 1 \mathrm{H}, J=16.0 \mathrm{~Hz}), 2.87(\mathrm{dd}, 1 \mathrm{H}, J=5.0,16.0 \mathrm{~Hz}), 3.24$ $(\mathrm{ddd}, 1 \mathrm{H}, J=7.0,7.5,9.0 \mathrm{~Hz}), 3.60(\mathrm{ddd}, 1 \mathrm{H}, J=7.0,8.0,9.0 \mathrm{~Hz}), 4.39(\mathrm{~m}, 2 \mathrm{H}), 4.65(\mathrm{~s}, 1 \mathrm{H}), 5.05(\mathrm{ddd}, 1 \mathrm{H}, J$ $=1.0,2.0,5.0 \mathrm{~Hz}), 6.02(\mathrm{~d}, 1 \mathrm{H}, J=5.5 \mathrm{~Hz}), 6.31(\mathrm{dd}, 1 \mathrm{H}, J=2.0,5.5 \mathrm{~Hz}) ;{ }^{13} \mathrm{C} \mathrm{NMR}\left(125 \mathrm{MHz} \mathrm{CDCl}_{3}\right) \delta$ 20.6, 42.4, 45.3, 62.4, 70.6, 78.0, 86.7, 134.3, 135.4, 159.2, 200.9; IR (thin film) $\mathrm{cm}^{-1} 2979 \mathrm{~s}, 2919 \mathrm{~s}, 1833 \mathrm{~m}$, 1722s, 1483s; mass spectrum (APCI): m/e (\% relative intensity) $224(100)(\mathrm{M}+1)^{+}, 206(35), 142$ (25), 137 (98), 102 (29); $t_{r}=23.5 \mathrm{~min}$ (minor), $29.9 \mathrm{~min}$ (major) (20\% $i$-PrOH in hexane).

\section{Compound 23-S (syn):}

$R_{f}=0.43[\mathrm{EtOAc}]$

${ }^{1} \mathrm{H}$ NMR $\left(300 \mathrm{MHz}, \mathrm{CDCl}_{3}\right) \delta 2.55(\mathrm{dd}, 1 \mathrm{H}, J=1.2,15.6 \mathrm{~Hz}), 3.00(\mathrm{dd}, 1 \mathrm{H}, J=5.4,15.6 \mathrm{~Hz}), 3.46(\mathrm{ddd}, 1 \mathrm{H}, J$ $=7.5,16.5 \mathrm{~Hz}), 3.66(\mathrm{ddd}, 1 \mathrm{H}, J=6.3,7.8,8.7 \mathrm{~Hz}), 3.86(\mathrm{~s}, 3 \mathrm{H}), 4.40(\mathrm{~m}, 2 \mathrm{H}), 4.97(\mathrm{~s}, 1 \mathrm{H}), 5.20(\mathrm{ddd}, 1 \mathrm{H}, J=$ 
$1.2,1.8,5.4 \mathrm{~Hz}), 6.38(\mathrm{~d}, 1 \mathrm{H}, J=6.0 \mathrm{~Hz}), 6.47(\mathrm{dd}, 1 \mathrm{H}, J=1.8,6.0 \mathrm{~Hz}) ;{ }^{13} \mathrm{C} \mathrm{NMR}\left(75 \mathrm{MHz}, \mathrm{CDCl}_{3}\right) \delta 42.2$, 45.4, 53.5, 62.8, 68.1 , 79.0, 88.3, 130.5, 136.3, 158.7, 167.2, 198.4; IR (thin film) $\mathrm{cm}^{-1} 3004 \mathrm{~m}, 2957 \mathrm{~m}, 2906 \mathrm{~m}$, 1738s, 1733s, 1674m; mass spectrum (APCI): m/e (\% relative intensity) $268(28)(\mathrm{M}+1)^{+}, 236(30), 142(51)$, 98 (36), 88 (100); $t_{r}=34.3 \min$ (major), $40.3 \min$ (minor) (20\% $i$-PrOH in hexane).

\section{Compound 24-S.}

$R_{f}=0.50[\mathrm{EtOAc}]$

${ }^{1} \mathrm{H}$ NMR $\left(300 \mathrm{MHz}, \mathrm{CDCl}_{3}\right) \delta 1.51(\mathrm{~s}, 3 \mathrm{H}), 1.52(\mathrm{~s}, 3 \mathrm{H}), 2.49(\mathrm{~d}, 1 \mathrm{H}, J=5.6 \mathrm{~Hz}), 2.68(\mathrm{~d}, 1 \mathrm{H}, J=15.6 \mathrm{~Hz})$, $3.22(\mathrm{ddd}, 1 \mathrm{H}, J=6.8,8.0,9.0 \mathrm{~Hz}), 3.59(\mathrm{ddd}, 1 \mathrm{H}, J=6.6,8.0,9.3 \mathrm{~Hz}), 4.38(\mathrm{~m}, 2 \mathrm{H}), 4.60(\mathrm{~s}, 1 \mathrm{H}), 5.94(\mathrm{~d}, 1 \mathrm{H}$, $J=5.7 \mathrm{~Hz}), 6.10(\mathrm{~d}, 1 \mathrm{H}, J=5.7 \mathrm{~Hz}) ;{ }^{13} \mathrm{C} \mathrm{NMR}\left(75 \mathrm{MHz}, \mathrm{CDCl}_{3}\right) \delta 20.9,23.1,42.3,51.1,62.4,69.4,84.6$, 86.6, 134.1, 138.5, 159.3, 201.3; IR (thin film) $\mathrm{cm}^{-1} 2978 \mathrm{~s}, 2932 \mathrm{~s}, 1748 \mathrm{~s}, 1733 \mathrm{~s}, 1622 \mathrm{~m}$; mass spectrum (APCI): m/e (\% relative intensity) $238(100)(\mathrm{M}+1)^{+}, 220(27), 151(23), 142(33), 61(72) ; \mathrm{t}_{\mathrm{r}}=17.3 \mathrm{~min}$ (major), $28.8 \mathrm{~min}$ (minor) (20\% $i$-PrOH in hexane).

\section{Compound 25-S (anti).}

$R_{f}=0.42[\mathrm{EtOAc}] ;[\alpha]_{\mathrm{D}}^{23}=+68.8\left(\mathrm{c}=0.25, \mathrm{CH}_{2} \mathrm{Cl}_{2}\right)$;

${ }^{1} \mathrm{H}$ NMR $\left(500 \mathrm{MHz}, \mathrm{CDCl}_{3}\right) \delta 1.84(\mathrm{~d}, 3 \mathrm{H}, J=1.5 \mathrm{~Hz}), 2.49(\mathrm{~d}, 1 \mathrm{H}, J=16.0 \mathrm{~Hz}), 2.84(\mathrm{dd}, 1 \mathrm{H}, J=5.0,16.0$ $\mathrm{Hz}$ ), 3.28 (ddd, 1H, $J=7.0,8.0,9.0 \mathrm{~Hz}), 3.66(\mathrm{ddd}, 1 \mathrm{H}, J=7.0,8.0,9.0 \mathrm{~Hz}), 4.33$ (ddd, 1H, $J=7.0,9.0,9.0$ $\mathrm{Hz}), 4.39$ (ddd, 1H, $J=7.0,8.5,9.0 \mathrm{~Hz}), 4.76(\mathrm{~d}, 1 \mathrm{H}, J=5.0 \mathrm{~Hz}), 4.82(\mathrm{~d}, 1 \mathrm{H}, J=4.5 \mathrm{~Hz}), 4.93(\mathrm{~m}, 1 \mathrm{H}), 5.80$ $(\mathrm{dq}, 1 \mathrm{H}, J=1.5,1.5 \mathrm{~Hz}) ;{ }^{13} \mathrm{C} \mathrm{NMR}\left(125 \mathrm{MHz}, \mathrm{CDCl}_{3}\right) \delta$ 12.9, 42.6, 44.8, 62.5, 64.8, 80.3, 80.8, 124.2, 146.6, 158.9, 201.1; IR (thin film) $\mathrm{cm}^{-1} 2960 \mathrm{~m}, 2918 \mathrm{~m}, 1746 \mathrm{~s}, 1721 \mathrm{~s}, 1422 \mathrm{~m}$; mass spectrum (APCI): m/e (\% relative intensity) $224(100)(\mathrm{M}+1)^{+}, 206(23), 142(26), 137(88), 100(17) ; t_{r}=22.9$ min (major), 26.2 min (minor) (20\% $i$-PrOH in hexane).

\section{Compound 26-S (anti).}

$R_{f}=0.51[\mathrm{EtOAc}]$

${ }^{1} \mathrm{H}$ NMR $\left(500 \mathrm{MHz}, \mathrm{CDCl}_{3}\right) \delta 2.71(\mathrm{~d}, 1 \mathrm{H}, J=16.0 \mathrm{~Hz}), 2.85(\mathrm{dd}, 1 \mathrm{H}, J=5.0,16.0 \mathrm{~Hz}), 3.27(\mathrm{ddd}, 1 \mathrm{H}, J=6.5$, 8.0, $9.0 \mathrm{~Hz}), 3.71(\mathrm{ddd}, 1 \mathrm{H}, J=6.5,8.0,9.0 \mathrm{~Hz}), 4.35(\mathrm{ddd}, 1 \mathrm{H}, J=7.0,9.0,9.0 \mathrm{~Hz}), 4.43(\mathrm{ddd}, 1 \mathrm{H}, J=6.5$, 8.0, 9.0 Hz), $4.83(\mathrm{~d}, 1 \mathrm{H}, J=5.0 \mathrm{~Hz}), 4.89(\mathrm{dd}, 1 \mathrm{H}, J=1.5,5.0 \mathrm{~Hz}), 5.03(\mathrm{dd}, 1 \mathrm{H}, J=2.0,4.5 \mathrm{~Hz}), 6.35(\mathrm{~d}, 1 \mathrm{H}$, $J=2.0 \mathrm{~Hz}) ;{ }^{13} \mathrm{C}$ NMR $\left(125 \mathrm{MHz}, \mathrm{CDCl}_{3}\right) \delta 42.6,44.1,62.6,64.3,81.2,81.8,126.5,129.9,158.7,199.6 ;$ IR (thin film) $\mathrm{cm}^{-1} 2964 \mathrm{~m}, 2917 \mathrm{~m}, 1770 \mathrm{~s}, 1714 \mathrm{~s}, 1599 \mathrm{~s}$; mass spectrum (EI): m/e (\% relative intensity) 290 (9) $\mathrm{M}^{+}+2,288$ (9) $\mathrm{M}^{+}, 174$ (15), 172 (15), 161 (8), 159 (8), 128 (40), 100 (100); $t_{r}=50.8$ min (major), 61.5 min (minor) (10\% $i$-PrOH in hexane). 


\section{Compound 27-S (syn).}

$R_{f}=0.63[\mathrm{EtOAc}]$

${ }^{1} \mathrm{H}$ NMR $\left(500 \mathrm{MHz}, \mathrm{CDCl}_{3}\right) \delta 2.48(\mathrm{~d}, 1 \mathrm{H}, J=13.5 \mathrm{~Hz}), 2.81(\mathrm{~m}, 1 \mathrm{H}), 2.88(\mathrm{dd}, 1 \mathrm{H}, J=5.0,13.5 \mathrm{~Hz}), 3.57$ (ddd, $1 \mathrm{H}, J=1.0,5.0,16.0 \mathrm{~Hz}), 3.79(\mathrm{ddd}, 1 \mathrm{H}, J=1.0,4.0,16.0 \mathrm{~Hz}), 4.06(\mathrm{~m}, 1 \mathrm{H}), 4.32(\mathrm{~d}, 1 \mathrm{H}, J=15.5 \mathrm{~Hz})$, $4.60(\mathrm{~d}, 1 \mathrm{H}, J=15.5 \mathrm{~Hz}), 4.78(\mathrm{~d}, 1 \mathrm{H}, J=5.0 \mathrm{~Hz}), 4.95(\mathrm{~d}, 1 \mathrm{H}, J=5.0 \mathrm{~Hz}), 5.03(\mathrm{~m}, 1 \mathrm{H}), 6.32(\mathrm{~m}, 1 \mathrm{H}), 7.38-$ $7.51(\mathrm{~m}, 9 \mathrm{H}), 7.59-7.70(\mathrm{~m}, 6 \mathrm{H}) ;{ }^{13} \mathrm{C} \mathrm{NMR}\left(75 \mathrm{MHz}, \mathrm{CDCl}_{3}\right) \delta$ 42.5, 45.3, 60.4, 62.6, 65.2, 77.7, 79.0, 128.0, $129.8,130.4,132.9,135.2,146.7,158.5,201.1$; IR (thin film) $\mathrm{cm}^{-1} 3066 \mathrm{~s}, 3010 \mathrm{~m}, 2911 \mathrm{~m}, 1777 \mathrm{w}, 1668 \mathrm{w}$, $1587 \mathrm{~m}$; mass spectrum (APCI): m/e (\% relative intensity) $498(19)(\mathrm{M}+1)^{+}, 420(100), 222(44), 138(19) ; t_{r}=$ $24.9 \mathrm{~min}$ (minor), $32.1 \mathrm{~min}$ (major) (10\% $i$-PrOH in hexane).

\section{Compound 27-S (anti).}

$R_{f}=0.59[\mathrm{EtOAc}] ;[\alpha]_{\mathrm{D}}^{23}=-21.3\left(\mathrm{c}=0.31, \mathrm{CH}_{2} \mathrm{Cl}_{2}\right)$;

${ }^{1} \mathrm{H}$ NMR $\left(500 \mathrm{MHz}, \mathrm{CDCl}_{3}\right) \delta 2.42(\mathrm{~d}, 1 \mathrm{H}, J=16.0 \mathrm{~Hz}), 2.81(\mathrm{dd}, 1 \mathrm{H}, J=5.0,16.0 \mathrm{~Hz}), 3.07(\mathrm{ddd}, 1 \mathrm{H}, J=6.5$, 7.0, 8.5 Hz), $3.49(\mathrm{ddd}, 1 \mathrm{H}, J=7.0,8.5,9.0 \mathrm{~Hz}), 4.25(\mathrm{ddd}, 1 \mathrm{H}, J=6.5,7.0,7.0 \mathrm{~Hz}), 4.34(\mathrm{ddd}, 1 \mathrm{H}, J=6.5$, 8.5, $9.0 \mathrm{~Hz}), 4.42(\mathrm{ddd}, 1 \mathrm{H}, J=2.0,2.0,15.0 \mathrm{~Hz}), 4.52(\mathrm{ddd}, 1 \mathrm{H}, J=2.0,2.0,15.0 \mathrm{~Hz}), 4.84(\mathrm{~d}, 1 \mathrm{H}, J=4.5$ $\mathrm{Hz}), 4.89(\mathrm{~d}, 1 \mathrm{H}, J=5.0 \mathrm{~Hz}), 4.97(\mathrm{~m}, 1 \mathrm{H}), 6.04(\mathrm{dt}, 1 \mathrm{H}, J=2.0,2.0 \mathrm{~Hz}), 7.38-7.66(\mathrm{~m}, 15 \mathrm{H}) ;{ }^{13} \mathrm{C} \mathrm{NMR}(75$ $\left.\mathrm{MHz}, \mathrm{CDCl}_{3}\right) \delta 42.2,45.3,59.5,62.4,64.6,77.9,79.9,124.4,128.0,130.3,133.1,135.1,150.1,158.9,200.7$; IR (thin film) $\mathrm{cm}^{-1} 3069 \mathrm{~m}, 3049 \mathrm{~m}, 2919 \mathrm{~m}, 1751 \mathrm{~s}, 1722 \mathrm{~s}, 1428 \mathrm{~m}$; mass spectrum (APCI): m/e (\% relative intensity) $498(27)(\mathrm{M}+1)^{+}, 420$ (100), 259 (11), 222 (33), 135 (25); $t_{r}=47.2$ min (major), 58.0 min (minor) (10\% $i-\mathrm{PrOH}$ in hexane).

\section{Reductive Cleavage of the Oxazolidinone Auxiliary.}

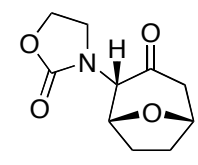

i-S

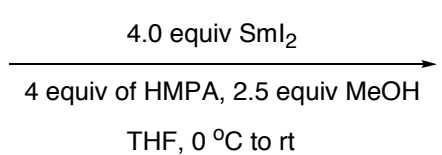

THF, $0{ }^{\circ} \mathrm{C}$ to $r t$

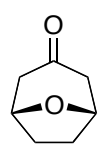

iv

To a stirred solution of $\mathbf{i}-S(20.0 \mathrm{mg}, 0.095 \mathrm{mmol})$ in $\mathrm{THF}(2 \mathrm{~mL})$ at $0{ }^{\circ} \mathrm{C}$ were added $\mathrm{MeOH}(7.5 \mathrm{mg}$, $0.234 \mathrm{mmol})$, and then $3.8 \mathrm{~mL}$ of a solution of $\mathrm{SmI}_{2}(0.38 \mathrm{mmol})$ and HMPA $(0.38 \mathrm{mmol})$ in the reaction mixture dropwise at $0{ }^{\circ} \mathrm{C}$. After $10 \mathrm{~min}$, the resulting solution was allowed to warm to room temperature. The reaction was quenched by the addition of a sat aq $\mathrm{NaHCO}_{3}$ solution $(5 \mathrm{~mL})$. The solution was filtered and the filtrate was washed with sat aq $\mathrm{Na}_{2} \mathrm{~S}_{2} \mathrm{O}_{3}$. After separation of organic and aqueous phases, the aqueous solution was extracted with $\mathrm{CH}_{2} \mathrm{Cl}_{2}$, and the combined organic extracts were washed by sat aq $\mathrm{NaCl}$, and then dried over $\mathrm{Na}_{2} \mathrm{SO}_{4}$. After removing the solvent carefully under reduced pressure, the residue was subjected to flash silica gel column chromatography [eluent: ether/pentane] to afford $7.0 \mathrm{mg}$ (59\% yield) of the desired product iv. 
$\mathrm{R}_{\mathrm{f}}=0.44$ [66\% EtOAc in Hexane];

${ }^{1} \mathrm{H}$ NMR $\left(500 \mathrm{MHz}, \mathrm{CDCl}_{3}\right) \delta$ 1.75-1.78 (m, 2H), 2.02-2.10 (m, 2H), $2.30(\mathrm{~d}, 2 \mathrm{H}, \mathrm{J}=15.5 \mathrm{~Hz}), 2.71(\mathrm{dd}, 2 \mathrm{H}, \mathrm{J}$ $=5.0,15.5 \mathrm{~Hz}), 4.71-4.75(\mathrm{~m}, 2 \mathrm{H}) ;{ }^{13} \mathrm{C} \mathrm{NMR}\left(125 \mathrm{MHz}, \mathrm{CDCl}_{3}\right) \delta 29.5,49.7,74.9,207.8$; IR (thin film) $\mathrm{cm}^{-1}$ 2959s, 2923s, 1721s, 1199m; exact mass calcd for $\mathrm{C}_{7} \mathrm{H}_{10} \mathrm{O}_{2}(\mathrm{M}+\mathrm{Na})^{+}: 149.0573$, found: 149.0572 . 